\title{
A Short Proof of a Kupershmidt-Wilson Theorem
}

\author{
L. A. Dickey \\ Leningradsky av. 28, fl. 59, SU-125040 Moscow, USSR
}

\begin{abstract}
This is a short elementary proof of a statement originally observed by Adler, then pursued by the author, by Kupershmidt and Wilson, and in a more general setting by Drinfeld and Sokolov.
\end{abstract}

Gardner, Zakharov, and Faddeev have found a Hamiltonian structure for the Korteweg-de Vries equation. Gelfand and the author constructed much more general Hamiltonian structures and integrable equations which were Hamiltonian in those structures. Adler [1] supposed that there is also a second Hamiltonian structure for those equations and gave without proof an expression for this second symplectic form. This conjecture was confirmed by Gelfand and the author [2]. Kupershmidt and Wilson [3] showed that "the second symplectic form" is equivalent to a very simple symplectic form of the Gardner-Zakharov-Miura type and this equivalence is given by a "general Miura transformation" introduced by these authors. This also follows from a recent paper of Drinfeld and Sokolov [4].

Since the theorem of Kupershmidt and Wilson is very important we think that it is useful to give a very simple proof of this theorem by a direct calculation.

We remind the reader the formulation of this theorem. Let $R$ be a ring of formal operators $\sum_{-\infty}^{m} a_{i} \partial^{i}$ where $a_{i}$ belong to a differential algebra $a$ and $\partial^{i}$ are symbols; the multiplication is defined by the rules

$$
\partial^{i} \partial^{j}=\partial^{i+j}, \quad \partial^{i} f=f \partial^{i}+\left(\begin{array}{l}
i \\
1
\end{array}\right) f^{\prime} \partial^{i-1}+\left(\begin{array}{l}
i \\
2
\end{array}\right) f^{\prime \prime} \partial^{i-2}+\ldots, \quad f \in a .
$$

Let $R_{+}$be a subring of "differential operators" $\sum_{0}^{m} a_{i} \partial^{i}$, and let $R_{-}$be a subring of "Volterra's integral operators," $\sum_{-\infty}^{-1} a_{i} \partial^{i}$. We denote

$$
\left(\sum_{-\infty}^{m} a_{i} \partial^{i}\right)_{+}=\sum_{0}^{m} a_{i} \partial^{i}, \quad\left(\sum_{-\infty}^{m} a_{i} \partial^{i}\right)_{-}=\sum_{-\infty}^{-1} a_{i} \partial^{i}, \quad \text { res } \sum_{-\infty}^{m} a_{i} \partial^{i}=a_{-1} .
$$


We define the integral $\tilde{f}=\int f d x$ in a formal sense as the projection $a \rightarrow \tilde{a}=a / a^{\prime}$; $\int f d x$ is an equivalence class of $f \in a$ modulo exact derivatives $g^{\prime}$, where $g \in a$. It is easy to show that $\int \operatorname{res} A B d x=\int \operatorname{res} B A d x ; A, B \in R$. Now we assume that $a$ consists of differential polynomials in $u_{i}$ which are the coefficients of a differential operator $L=\sum_{0}^{n} u_{i} \partial^{i}, u_{n}=1$. For $\tilde{f} \in \tilde{a}$ let $X_{f}$ denote $\sum_{0}^{n-1} \partial^{-i-1} \delta f / \delta u_{i}\left[\right.$ where $\delta f / \delta u_{i}$ $=\sum_{0}^{\infty}(-d / d x)^{k} \partial f / \partial u_{i}^{(k)}$ are variational derivatives]. The Poisson bracket of the second kind is defined as

$$
\{\tilde{f}, \tilde{g}\}=\int \operatorname{res}\left\{L\left(X_{f} L\right)_{+}-\left(L X_{f}\right) L\right\} X_{g} d x .
$$

If $L$ is written in a multiplicative form

$$
L=\sum_{0}^{n} u_{i} \partial^{i}=\left(\partial-v_{n}\right)\left(\partial-v_{n-1}\right) \ldots\left(\partial-v_{1}\right)
$$

then $u_{i}$ will be expressed in terms of $v_{i}$. This will be called the "Miura transformation." Note that $v_{i}$ do not belong to $a$. If $a_{v}$ is a differential algebra generated by $\left\{v_{i}\right\}$ the Miura transformation specifies an imbedding $a$ in $a_{v}$.

\section{Theorem.}

$$
\{\tilde{f}, \tilde{g}\}=-\int \sum_{i=1}^{n} \frac{\delta f}{\delta v_{i}}\left(\frac{\delta g}{\delta v_{i}}\right)^{\prime} d x .
$$

Proof. The expression of $\delta f / \delta v_{i}$ in terms of $\delta f / \delta u_{i}$ can be obtained from

$$
\delta \tilde{f}=\int \sum_{0}^{n-1} \frac{\delta f}{\delta u_{i}} \delta u_{i} d x=\int \sum_{1}^{n} \frac{\delta f}{\delta v_{i}} \delta v_{i} d x .
$$

We have $\int \sum \delta f / \delta u_{i} \cdot \delta u_{i} d x=\int \operatorname{res} X_{f} \delta L d x$. Denoting $\partial-v_{i}=\partial_{i}$ we obtain from (2) an equality

$$
\begin{aligned}
\int \operatorname{res} X_{f} \delta L d x & =-\int \operatorname{res} X_{f} \sum_{i} \partial_{n} \ldots \partial_{i+1} \delta v_{2} \partial_{i-1} \ldots \partial_{1} d x \\
& =-\int \operatorname{res} \sum_{i} \delta v_{i} \partial_{i-1} \ldots \partial_{1} X_{f} \partial_{n} \ldots \partial_{i+1} d x
\end{aligned}
$$

whence

$$
\frac{\delta f}{\delta v_{i}}=-\operatorname{res} \partial_{i-1} \ldots \partial_{1} X_{f} \partial_{n} \ldots \partial_{i+1}
$$

Now

$$
\begin{aligned}
\int \sum \frac{\delta f}{\delta v_{i}}\left(\frac{\delta g}{\delta v_{i}}\right)^{\prime} d x & =\int \sum \operatorname{res}\left(\partial_{i-1} \ldots X_{f} \ldots \partial_{i+1}\right)\left(\operatorname{res}\left(\partial_{i-1} \ldots X_{g} \ldots \partial_{i+1}\right)\right) d x \\
& =\int \operatorname{res}\left\{\sum \partial_{i-1} \ldots X_{f} \ldots \partial_{i+1}\left[\partial, \operatorname{res}\left(\partial_{i-1} \ldots X_{g} \ldots \partial_{i+1}\right)\right]\right\} d x
\end{aligned}
$$

We can replace here $\partial$ by $\partial_{i}$. From the obvious relations

$$
\operatorname{res} A=\left(\partial A_{-}\right)_{+}=\left(A_{-} \partial\right)_{+}=\left(\partial_{i} A_{-}\right)_{+}=\left(A_{-} \partial_{i}\right)_{+}
$$


we obtain

$$
\begin{aligned}
\int \sum \frac{\delta f}{\delta v_{i}}\left(\frac{\delta g}{\delta v_{i}}\right)^{\prime} d x= & \int \operatorname{res}\left\{\sum \partial_{i-1} \ldots X_{f} \ldots \partial_{i}\left(\left(\partial_{i-1} \ldots X_{g} \ldots \partial_{i+1}\right)_{-} \partial_{i}\right)_{+}\right. \\
& \left.-\sum \partial_{i} \ldots X_{f} \ldots \partial_{i+1}\left(\partial_{i}\left(\partial_{i-1} \ldots X_{g} \ldots \partial_{i+1}\right)_{-}\right)_{+}\right\} d x .
\end{aligned}
$$

The subscripts - can be omitted : if we replace them by + , the right-hand side will be zero (in this case external subscripts + can be dropped and both the terms mutually cancel). We get

$$
\begin{aligned}
\int \sum \frac{\delta f}{\delta v_{i}}\left(\frac{\delta g}{\delta v_{i}}\right)^{\prime} d x= & \int \operatorname{res}\left\{\sum \partial_{i-1} \ldots X_{f} \ldots \partial_{i}\left(\partial_{i-1} \ldots X_{g} \ldots \partial_{i}\right)_{+}\right. \\
& \left.-\sum \partial_{i} \ldots X_{f} \ldots \partial_{i+1}\left(\partial_{i} \ldots X_{g} \ldots \partial_{i+1}\right)_{+}\right\} d x \\
= & \int \operatorname{res}\left\{X_{f} \partial_{n} \ldots \partial_{1}\left(X_{g} \partial_{n} \ldots \partial_{1}\right)_{+}-\partial_{n} \ldots \partial_{1} X_{f}\left(\partial_{n} \ldots \partial_{1} X_{g}\right)_{+}\right\} d x \\
= & \int \operatorname{res}\left\{X_{f} L\left(X_{g} L\right)_{+}-L X_{f}\left(L X_{g}\right)_{+}\right\} d x \\
= & \int \operatorname{res}\left\{L\left(X_{g} L\right)_{+}-\left(L X_{g}\right)_{+} L\right\} X_{f} d x=\{g, f\}
\end{aligned}
$$

as stated.

Remark. We could restrict ourselves by operators $L$ with $u_{n-1}=0$. In this case $X_{f}=\sum_{0}^{n-1} X_{i} \partial^{-i-1}$, where $X_{i}=\delta f / \delta u_{i}$ only for $i \leqq n-2$. Here $X_{n-1}$ is determined from $\operatorname{res}\left[L, X_{f}\right]=0$ (see [2]). Thus $v_{i}$ are no longer independent, $\sum v_{i}=0$. We define $\delta f / \delta v_{i}$ to be such $a_{i}$ that $\delta \tilde{f}=\int a_{i} \delta v_{i} d x$ and $\sum a_{i}=0$. Then the theorem remains valid. It is only necessary to prove that the sum of right-hand side of (4) is zero. It is sufficient to prove that the derivative of this sum is zero, which can be done by analogy with the proof of the above theorem.

\section{References}

1. Adler, M.: Invent. Math. 50, 219-248 (1979)

2. Gelfand, I.M., Dickey, L.A.: The family of Hamiltonian structures connected with integrable nonlinear equations. Preprint of the Inst. Appl. Math. 136, 1-41 (1978)

3. Kupershmidt, B.A., Wilson, G.W.: Invent. Math. 62, 403-436 (1981)

4. Drinfeld, V.G., Sokolov, V.V.: Dokl. Akad. Nauk USSR 258, 11-16 (1981) (in Russian)

Communicated by A. Jaffe

Received January 12, 1982 
